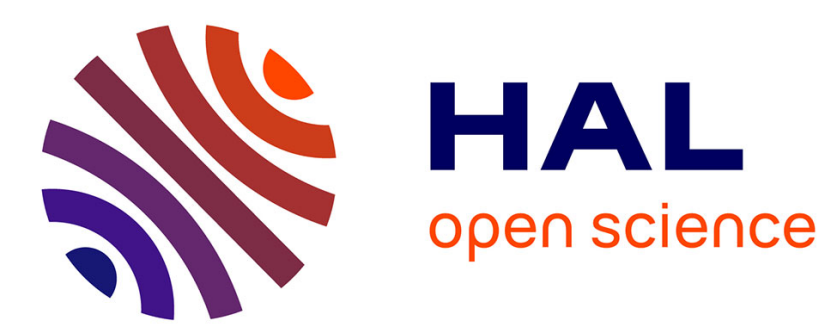

\title{
Active control of thermoacoustic amplification in an annular engine
}

Cyril Desjouy, Guillaume Penelet, Pierrick Lotton

\section{To cite this version:}

Cyril Desjouy, Guillaume Penelet, Pierrick Lotton. Active control of thermoacoustic amplification in an annular engine. Journal of Applied Physics, 2010, 108 (11), pp.114904. 10.1063/1.3512962 . hal-02057432

\section{HAL Id: hal-02057432 \\ https://hal-univ-lemans.archives-ouvertes.fr/hal-02057432}

Submitted on 5 Mar 2019

HAL is a multi-disciplinary open access archive for the deposit and dissemination of scientific research documents, whether they are published or not. The documents may come from teaching and research institutions in France or abroad, or from public or private research centers.
L'archive ouverte pluridisciplinaire HAL, est destinée au dépôt et à la diffusion de documents scientifiques de niveau recherche, publiés ou non, émanant des établissements d'enseignement et de recherche français ou étrangers, des laboratoires publics ou privés. 


\title{
Active control of thermoacoustic amplification in an annular engine
}

\author{
C. Desjouy, a) G. Penelet, and P. Lotton \\ Laboratoire d'Acoustique de l'Université du Maine, UMR CNRS 6613, Avenue Olivier Messiaen, \\ 72085 Le Mans Cedex 9, France
}

(Received 29 June 2010; accepted 3 October 2010; published online 7 December 2010)

\begin{abstract}
In this paper, a new method is proposed to control the thermoacoustic amplification in thermoacoustic engines. This method, based on the active control of the spatial distribution of the acoustic field by means of auxiliary acoustic sources, is applied here to an annular thermoacoustic engine. Two auxiliary acoustic sources are used to tune the spatial distribution of the sound field in the engine in such a way that the thermal-to-acoustic energy conversion occurring into the thermoacoustic core is maximized. An experimental study of this device is proposed, which should be considered as a proof-of-concept study, aiming at demonstrating that the addition of auxiliary acoustic sources can be used advantageously to improve the efficiency of thermoacoustic engines. The overall device is characterized below and above the onset of thermoacoustic instability. It is demonstrated that below the onset of thermoacoustic instability, there exists an optimum phase shift between the auxiliary sources which maximizes the acoustic power available in the annular waveguide. When the device is operated above the onset of thermoacoustic instability, it is demonstrated that the appropriate tuning of the two auxiliary sources enables to improve significantly the acoustic work produced into the engine (compared to the case without active control), that the additional output acoustic power is significantly larger than the input electric power supplied to the acoustic sources, and that the overall efficiency of the engine is thus significantly increased. A discussion about the applicability of this new method for the improvement of actual, high power thermoacoustic engines is also provided. (c) 2010 American Institute of Physics.

[doi:10.1063/1.3512962]
\end{abstract}

\section{INTRODUCTION}

For the transformation of thermal energy into mechanical energy, thermoacoustic engines employ the interaction between a gas column and an inhomogeneously heated porous solid, which results in the onset of self-excited acoustic oscillations. These engines have been studied for several decades $^{1}$ because they may find industrial applications for instance as a source of mechanical work for pulse tube refrigerators, or for the production of electricity. ${ }^{2}$ An important breakthrough in the development of thermoacoustic engines was realized ten years ago by Backhaus et $a{ }^{3}{ }^{3}$ when they built a so-called thermoacoustic stirling heat engine (TASHE) able to provide $700 \mathrm{~W}$ of acoustic power with $30 \%$ efficiency. This thermoacoustic engine notably employs a closed-loop waveguide arrangement to provide the adequate traveling wave phasing of the acoustic wave within the porous solid where the thermal to acoustical energy conversion occurs.

The physical principles of the thermoacoustic amplification process are nowadays well-established and the linear thermoacoustic theory provides an effective tool for predicting the critical temperature gradient corresponding to the onset of the thermoacoustic instability ${ }^{1}$ (i.e., the beginning of wave amplitude growth) in various thermoacoustic devices. However, in practical devices where the sound wave reaches very large amplitudes, the linear thermoacoustic theory fails to predict accurately the steady-state conditions (in terms of

${ }^{a)}$ Electronic mail: cyril.desjouy@univ-lemans.fr. acoustic pressure amplitude and temperature distribution along the thermoacoustic core) which are controlled by several nonlinear effects. Among the nonlinear effects saturating the wave amplitude growth, some classical effects are involved, like the cascade process of higher harmonics generation $^{4,5}$ or the minor losses due to aerodynamical edge effects through the porous solid and the heat exchangers ${ }^{6}$ but there are also less common effects which impact the temperature gradient along the thermoacoustic core, like the acoustically enhanced thermal diffusivity of the solid, ${ }^{7}$ and the forced convection due to the generation of acoustic streaming. ${ }^{8,9}$ An important research has been devoted to the theoretical description of these effects ${ }^{10-14}$ and to the understanding of their influence on the saturation of the wave amplitude growth, ${ }^{4,15-18}$ but these studies are yet ineffectual to provide quantitative predictions of the performances of actual engines. In practice, thermoacoustic devices are generally designed with the help of available softwares based on linear (or weakly nonlinear) thermoacoustic theory, ${ }^{19}$ and the nonlinear effects are controlled (almost empirically) by using some passive elements judiciously placed inside the engine. For instance, the effects of flow separation due to geometrical singularities can be minimized by using flow straighteners, ${ }^{2,3}$ while acoustic streaming can be controlled using tapered tubes, ${ }^{3,20}$ jet pumps, ${ }^{2,3,21}$ or membranes. ${ }^{21}$

An important point concerns the control of acoustic streaming because this second-order time-averaged mass flux tends to reduce the temperature gradient along the thermoacoustic core, but also because it modifies the shape of the temperature distribution so that the thermoacoustic amplifi- 
cation process is also modified. ${ }^{17}$ More precisely, it has been demonstrated $^{22}$ that changing the shape of the temperature profile through the thermoacoustic core significantly modifies the acoustic impedance (i.e., the ratio of acoustic pressure amplitude to acoustic velocity amplitude) and the phase shift between pressure and velocity fluctuations in the porous solid. These modifications of the acoustic field, in turn, imply a modification of the thermodynamic cycle followed by one fluid parcel oscillating through the porous solid, with subsequent modifications of the thermoacoustic amplification process. This means that, in any thermoacoustic engine, there exists an optimum temperature distribution which allows to maximize the thermoacoustic amplification process by tuning the acoustic field inside the thermoacoustic core in the appropriate way. This also means that in an actual engine it is hardly probable that, after the onset of the thermoacoustic instability and when the steady state is reached, the temperature distribution along the thermoacoustic core corresponds exactly to the ideal one. However, one may find a technical solution to control the spatial distribution of the acoustic field (instead of controlling the temperature field) in order to optimize the process of thermoacoustic amplification, and this is the aim of the present works.

In this paper, a method of control is proposed to optimize the thermoacoustic amplification process in a thermoacoustic engine. This control (here called "active" to denote that, unlike passive methods, it requires to supply additional energy into the system in order to control its performances) is applied to an annular thermoacoustic engine, for which the presence of a closed-loop waveguide allows an easy control of the acoustic field by means of two acoustic transducers appropriately tuned in terms of amplitude, frequency and relative phasing. ${ }^{23}$ An experimental study is undertaken to investigate the possibility to tune the acoustic field inside the thermoacoustic core, by two loudspeakers, so that the thermoacoustic amplification process is maximized and the overall efficiency of the thermoacoustic engine (accounting for the electrical energy provided to the loudspeakers) is larger than its initial value without active control.

In Sec. II, the experimental apparatus is briefly presented, and attention is also focused on the definition and the measurement of its efficiency. Section III is devoted to the experimental characterization of the thermoacoustic amplification below the onset of thermoacoustic instability, when the acoustic field is sustained by the auxiliary sources. Then, in Sec. IV, the thermoacoustic system is characterized above the onset of the thermoacoustic instability, and it is shown that when the active control of sound is operated, the efficiency of the engine grows significantly. Finally, a discussion is provided in Sec. V, which aims at emphasizing the major points which should be elucidated before to conclude that active control of sound can be used advantageously in an actual, high power thermoacoustic engine.

\section{EXPERIMENTAL APPARATUS}

A schematic diagram of the experimental apparatus is shown in Fig. 1. The thermoacoustic core is a stainless steel tube of inner radius $R_{\mathrm{w}}=26.5 \mathrm{~mm}$ and length $L_{1}=0.53 \mathrm{~m}$.

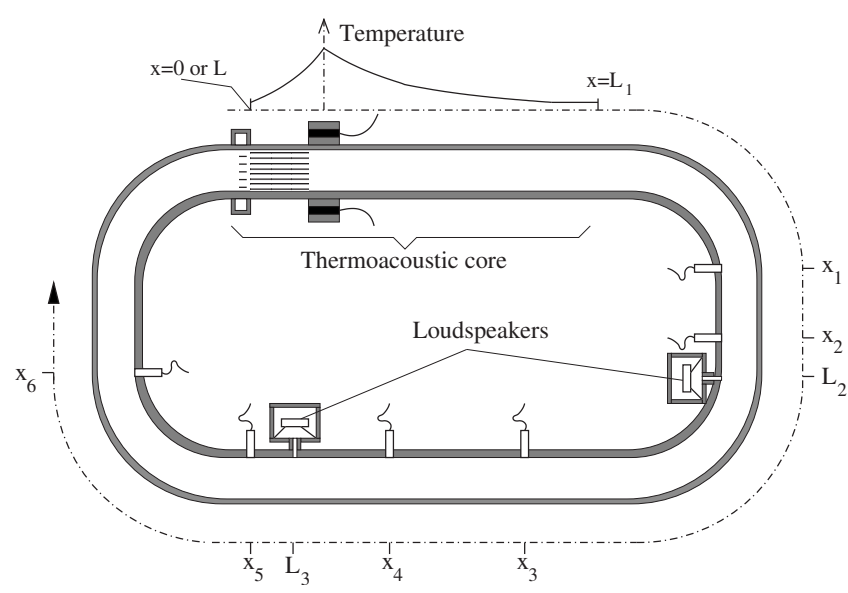

FIG. 1. Schematic diagram of the experimental apparatus. The spatial positions of the six microphones are $x_{1}=0.575 \mathrm{~m}, x_{2}=0.764 \mathrm{~m}, x_{3}=1.162 \mathrm{~m}$, $x_{4}=1.344 \mathrm{~m}, x_{5}=1.612 \mathrm{~m}, x_{6}=1.866 \mathrm{~m}$, and the positions of the auxiliary sources are $L_{2}=0.91 \mathrm{~m}$ and $L_{2}=1.47 \mathrm{~m}$.

The thermoacoustic amplification process occurs inside a ceramic porous material $(5 \mathrm{~cm}$ in length) with square channels of cross-section $0.9 \times 0.9 \mathrm{~mm}^{2}$. Note that this element is usually called "stack" by the community, in contrast with the term "regenerator" employed when the pores are sufficiently thin to ensure a quasi-isothermal contact between the solid walls and the oscillating fluid [as in the TASHE (Ref. 3)]. At the left side of the stack is the cold heat exchanger which consists of a copper circular shell set around the tube with flowing cooling water inside at room temperature, and a copper wire mesh inside the tube (attached to the left side of stack). At the right side of the stack is the hot heat exchanger: it is composed of eight electrical heat resistances connected to a thyristor unit and soldered in a stainless steel block set at the right side of the stack around the outer perimeter of the tube (Fig. 1). This hot heat exchanger allows to impose a strong temperature gradient along the stack, so that when this temperature gradient exceeds some critical value, the onset of the thermoacoustic instability results in the self-excitation of high amplitude acoustic waves at frequency $f_{\text {onset }} \approx 158 \mathrm{~Hz}$ which corresponds to the resonant frequency of the overall thermoacoustic system (including the auxiliary acoustic sources). The rest of the resonator (i.e., from $x=L_{1}$ to $x=L$ ) is made of three plexiglass tubes of inner radius $R_{\mathrm{w}}=26.5 \mathrm{~mm}$, namely two bended tubes $(56 \mathrm{~cm}$ in length, $14 \mathrm{~cm}$ in radius of curvature) and a straight tube (53 $\mathrm{cm}$ in length), which are connected to the thermoacoustic core in order to form the complete closed-loop waveguide. The overall unwrapped length of this torus shaped waveguide is $L=2.18 \mathrm{~m}$. The waveguide is filled with air at atmospheric pressure.

The two auxiliary acoustic sources are placed along the waveguide, at positions $L_{2}=0.91 \mathrm{~m}$ and $L_{3}=1.47 \mathrm{~m}$, respectively. Each of these sources is an electrodynamic loudspeaker (model Audax HP $170 \mathrm{MO}$ ) loaded by a rear enclosure of volume $V \simeq 6.4 \times 10^{-3} \mathrm{~m}^{3}$, and connected to the waveguide through a cylindrical tube of circular cross section (radius $r_{0}=3 \mathrm{~mm}$, length $l_{0}=30 \mathrm{~mm}$ ). The loudspeakers are connected through an audioamplifier to a dual channel signal generator (Tektronix AFG3029B) enabling to tune the 
frequency, amplitude and phase of the sinusoidal voltage applied to each of the loudspeakers. Note that the placement of the acoustic sources, separated by a distance $L_{2}-L_{1} \approx L / 4$, has not been chosen aimlessly: it has been demonstrated ${ }^{23,24}$ that in the case of a closed-loop resonator of unwrapped length $L$, it is possible, by means of two acoustic sources separated by a distance $L / 4$ and operating at frequency $f_{0}$ $=c_{0} / L$ (where $c_{0}$ stands for the sound velocity), to sustain an acoustic field which have the characteristics of a traveling wave (if the drivers are $\pi / 2$ out of phase), a standing wave (if the drivers are in phase), or any intermediate situation (in terms of standing/traveling wave component). In the present case, it is not possible to sustain a purely traveling wave due to the acoustic scattering induced by the thermoacoustic core, but there is still a possibility to control the traveling/ standing wave components of the acoustic field with an appropriate tuning of the excitation.

The instrumentation of the device is schematically shown in Fig. 1. The temperature measurement along the stack is carried out with five type $\mathrm{K}$ thermocouples $(70 \mu \mathrm{m}$ in diameter) equally spaced along the median axis of the stack and glued with a ceramic putty. Additional type $\mathrm{K}$ probes (1 $\mathrm{mm}$ in diameter) are also placed along the waveguide to provide information on the heat transfer due to acoustic streaming. Moreover, in order to characterize the acoustic field in the cold part of the resonator (i.e. from $x$ $=L_{1}$ to $x=L$ ) six microphones (model Bruel \& Kjaer 4136) are flush-mounted along the resonator. More precisely, a couple of microphones is placed in each of the three regions delimited by $x \in\left[L_{1}, L_{2}\right], x \in\left[L_{2}, L_{3}\right]$, and $x \in\left[L_{3}, L\right]$, respectively, and the two-microphone method ${ }^{25}$ is used in each region to compute the spatial distribution of the acoustic pressure and the acoustic volume velocity in the entire region $x$ $\in\left[L_{1}, L\right]$. Assuming the propagation of plane acoustic waves into the waveguide, the complex amplitude $\tilde{p}(x)$ of the acoustic pressure fluctuations $\left[p(x, t)=\mathfrak{R}\left\{\tilde{p}(x) e^{-i \omega t}\right\}\right.$, where $\omega=2 \pi f$ stands for the angular frequency of acoustic oscillations and $\mathfrak{R}$ denotes the real part of a complex number] in each of the three regions can be separated into two counterpropagating acoustic waves as follows:

$$
\tilde{p}(x)=\tilde{p}^{+}\left(x_{0}\right) e^{i k_{w}\left(x-x_{0}\right)}+\tilde{p}^{-}\left(x_{0}\right) e^{-i k_{w}\left(x-x_{0}\right)},
$$

where $x_{0}$ is an arbitrary position along the considered region, and where the complex wave number

$$
k_{w}=\frac{\omega}{c_{0}} \sqrt{1+\frac{f_{\nu}+(\gamma-1) f_{\kappa}}{1-f_{\nu}}},
$$

accounts for the viscous and thermal dissipation in the vicinity of the resonator's walls. In Eq. (2), $\gamma$ is the specific heat ratio, and the functions $f_{\nu}$ and $f_{\kappa}$ characterize the viscous and thermal coupling between the oscillating fluid and the cylindrical tube of radius $R_{w}{ }^{26}$ It is quite direct from Eq. (1) that measuring the phase and modulus of the complex amplitudes of acoustic pressure $\tilde{p}\left(x_{i, j}\right)$ at two positions $x_{i}$ and $x_{j}$ enables to calculate the complex amplitudes $\tilde{p}^{+}\left(x_{0}\right)$ and $\tilde{p}^{-}\left(x_{0}\right)$ from the measured $\tilde{p}\left(x_{i, j}\right)$ and thus to calculate the spatial distribution of acoustic pressure in each of the three regions $x$ $\in\left[L_{1}, L_{2}\right], x \in\left[L_{2}, L_{3}\right]$, and $x \in\left[L_{3}, L\right]$, using the three couples of microphones. The spatial distribution of acoustic volume velocity $\tilde{u}(x)$ can then be calculated from the distribution of acoustic pressure as follows: ${ }^{23}$

$$
\tilde{u}(x)=\frac{\pi R_{w}^{2}\left(1-f_{\nu}\right)}{i \omega \rho_{0}} \frac{\partial \tilde{p}}{\partial x} .
$$

Note that the two-microphone method used here to calculate the acoustic field is an indirect method which has to be undertaken with care in order to avoid large errors. ${ }^{25,27}$ In particular, a relative calibration of the six microphones has been made preliminarily by exposing them to the same sound field $^{28}$ in the frequency range of interest $(f$ $\in[150 \mathrm{~Hz}, 200 \mathrm{~Hz}])$, in order to prevent from large errors due to phase shifts between the sensors (inherent to the sensors themselves).

From the distribution of acoustic pressure and acoustic volume velocity it is also possible to calculate the distributions of the active acoustic power $\mathcal{I}(x)=(1 / 2) \mathfrak{R}\left\{\tilde{p}(x) \widetilde{u}^{*}(x)\right\}$ and of the reactive acoustic power $\mathcal{J}(x)=(1 / 2) \mathfrak{J}\left\{\widetilde{p}(x) \widetilde{u}^{*}(x)\right\}$ along the cold part of the annular resonator $\left(x \in\left[L_{1}, L\right]\right)$. These powers are used here to define some efficiency of the thermoacoustic engine. It is noticeable that the present engine is not a realistic "engine" because the acoustic work is not consumed by an acoustic load (as for instance an alternator $^{2}$ which would convert acoustic work into electricity). However, both $\mathcal{I}$ and $\mathcal{J}$ are representative of some heat flux converted into acoustic power: the active acoustic power $\mathcal{I}$ represents the power transported by the traveling wave component of the acoustic wave which propagates along the closed-loop waveguide, while the power $\mathcal{J}$ represents the standing wave component of the acoustic power. The efficiency $\eta$ of the engine is here defined as the ratio of the average acoustic power $\mathcal{W}_{a c}$ available in the cold part of the waveguide, defined as

$$
\mathcal{W}_{a c}=\frac{1}{L-L_{1}} \int_{L_{1}}^{L}|\mathcal{I}(x)+i \mathcal{J}(x)| d x,
$$

to the power $\mathcal{Q}_{h}$ provided to the electrical heat resistances,

$$
\eta_{0}=\frac{\mathcal{W}_{a c}}{\mathcal{Q}_{h}}
$$

\section{EXPERIMENTAL STUDY OF THE ENGINE BELOW THE ONSET OF THERMOACOUSTIC INSTABILITY}

In this section, the influence of the auxiliary sources on the acoustic field (in terms of amplitude and spatial distribution) is analyzed below the onset of thermoacoustic instability. In such conditions, the thermoacoustic process occurring into the stack does not lead to the onset of self-sustained thermoacoustic oscillations, but the acoustic field is sustained by the two auxiliary sources only.

The first step in the experiments consists in measuring the electrical power $\mathcal{Q}_{h}$ which must be supplied to the heat resistances in order to reach the onset of thermoacoustic instability (the auxiliary acoustic sources are, at this stage, switched off). The obtained electrical power is $\mathcal{Q}_{\text {onset }}$ $=347 \mathrm{~W}$, corresponding to a temperature difference $\Delta T_{\text {onset }}$ 
TABLE I. Resonant frequency $f$ and associated quality factor $Q$ with respect to the heating electrical power $\mathcal{Q}_{h}$.

\begin{tabular}{ccc}
\hline \hline $\mathcal{Q}_{h}$ & $f$ & \\
$(\mathrm{~W})$ & $(\mathrm{Hz})$ & $Q$ \\
\hline 0 & $157.50 \pm 0.05$ & $44.3 \pm 0.1$ \\
192 & $158.00 \pm 0.05$ & $59.6 \pm 0.1$ \\
338 & $159.20 \pm 0.05$ & $106.0 \pm 0.1$ \\
\hline \hline
\end{tabular}

$=139 \mathrm{~K}$ between both ends of the stack. The frequency of the self-sustained thermoacoustic oscillations is $f_{\text {onset }}$ $\simeq 160 \mathrm{~Hz}$. It is worth noting that these onset conditions are obtained when studying the overall device including the two auxiliary sources. Indeed, when the acoustic sources are removed and replaced by a rigid wall, the electrical power $\mathcal{Q}_{\text {onset }}$ is $310 \mathrm{~W}$, with a corresponding temperature difference $\Delta T_{\text {onset }}=119 \mathrm{~K}$ and an acoustic frequency $f_{\text {onset }} \simeq 155 \mathrm{~Hz}$. This means that the coupling between the waveguide and the auxiliary sources impacts the onset of thermoacoustic instability (notably because of the additional losses in the acoustic sources performing as acoustic loads). The impact of this coupling on the onset conditions has also been confirmed experimentally by modifying the coupling layout. The connecting tube of inner radius $r_{0}=3 \mathrm{~mm}$ has been replaced by a connecting tube of inner radius $r_{0}=8 \mathrm{~mm}$. In that case, the onset heating power supply increases up to $510 \mathrm{~W}$, with a corresponding $\Delta T_{\text {onset }}=186 \mathrm{~K}$ and an acoustic frequency $f_{\text {onset }} \simeq 172 \mathrm{~Hz}$.

As a second step, the frequency response function (i.e. the ratio, in the frequency domain, between the acoustic pressure at location $x=x_{1}$ and the electrical voltage supplied to the loudspeaker located at $x=L_{2}$ ) of the device is measured using an audio spectrum analyzer (Stanford research SR780) below the onset of thermoacoustic instability in the frequency range $[150 \mathrm{~Hz} ; 180 \mathrm{~Hz}]$. Table I reports the results obtained for the resonant frequency $f$ and its corresponding quality factor $Q$, and for three different values of the heating power supply $\mathcal{Q}_{h}$ below $\mathcal{Q}_{\text {onset }}$. It appears that both the resonant frequency and the quality factor are increasing with respect to $\mathcal{Q}_{h}$. The resonant frequency $f$ remains close to the natural frequency $f_{0}=c_{0} / L \simeq 157.8 \mathrm{~Hz}$ of the annular waveguide (where $c_{0} \simeq 344 \mathrm{~m} \mathrm{~s}^{-1}$ is the adiabatic sound speed at room temperature). Its evolution with respect to $\mathcal{Q}_{h}$ is due both to the dependence of the sound velocity on temperature and to the modification of the acoustic scattering properties of the thermoacoustic core induced by the modification of the temperature field with $\mathcal{Q}_{h}$. Moreover, the increase in the quality factor with respect to $\mathcal{Q}_{h}$ complies with our expectations: as $\mathcal{Q}_{h}$ is increased, the viscous and thermal losses in the device are gradually compensated by the thermoacoustic amplification occurring into the stack, which results in the increase in the quality factor $Q[Q$ should even tend toward infinity when $\mathcal{Q}_{h}$ approaches $\mathcal{Q}_{\text {onset }}$ (Ref. 29)].

Finally, the third step consists in measuring the average acoustic power $\mathcal{W}_{a c}$ available in the cold part of the waveguide $\left(x \in\left[L_{1} ; L\right]\right)$ for different values of $\mathcal{Q}_{h}$ below $\mathcal{Q}_{\text {onset }}$, and to investigate how the relative phasing of the auxiliary sources may control this acoustic power. In the experiments,

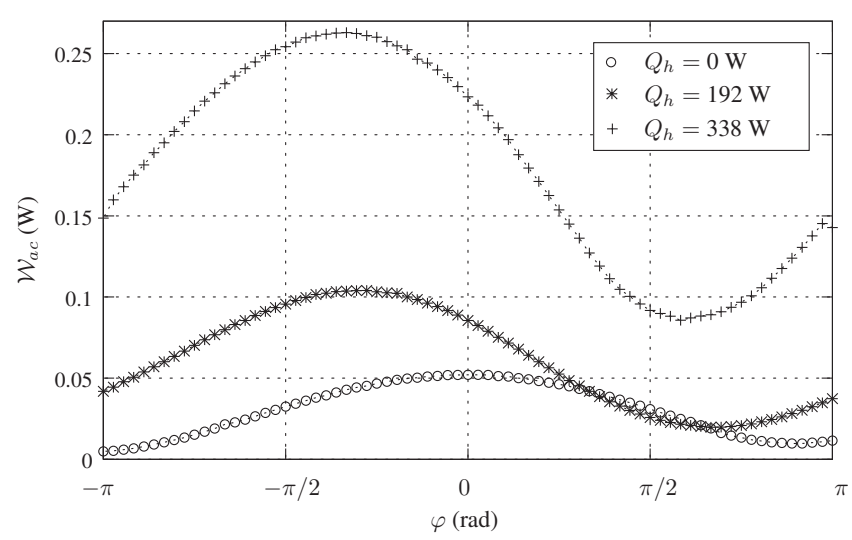

FIG. 2. Acoustic power $\mathcal{W}_{a c}$ available in the cold part of the waveguide with respect to the phase shift $\varphi$ between the signals supplied to the loudspeakers

the two auxiliary sources are supplied with the same electrical voltage $U=1.3 \mathrm{~V}_{\text {rms }}$ at resonant frequency $f$ (which depends on $\mathcal{Q}_{h}$ according to Table I). The spatial distribution of the acoustic field is determined from the measured complex acoustic pressures $\widetilde{p}\left(x_{i}\right)$ (with an accuracy of about $1.3 \%$ for the amplitude and $0.5^{\circ}$ for the phase) using the method described in Sec. II, allowing next to compute the average acoustic power $\mathcal{W}_{a c}$. Figure 2 reports this acoustic power $\mathcal{W}_{a c}$ with respect to the phase shift $\varphi$ between the harmonic signals supplied to the auxiliary sources, and for three different values of $\mathcal{Q}_{h}$. When $\mathcal{Q}_{h}=0 \mathrm{~W}$, the maximum of the acoustic power is obtained for $\varphi=0$. When $\mathcal{Q}_{h}$ increases, the optimum phase shift $\varphi$ varies from 0 to $-2 \pi / 7$ for $\mathcal{Q}_{h}$ $=192 \mathrm{~W}$, and to $-\pi / 3$ for $\mathcal{Q}_{h}=338 \mathrm{~W}$ (just below the threshold of thermoacoustic instability). Consequently, there exists an optimum phase shift $\varphi$ between the auxiliary sources, which depends on the temperature distribution in the thermoacoustic core, and which allows to maximize the available acoustic power $\mathcal{W}_{a c}$.

Two spatial distributions of the acoustic field are reported in Fig. 3 when setting the heating power supply to 192 W. The first distribution corresponds to an "advantageous" case for which the acoustic power $\mathcal{W}_{a c}$ is maximum ( $\varphi=-2 \pi / 7$, see Fig. 2, continuous lines) and the second one to a "disadvantageous" case for which $\mathcal{W}_{a c}$ is minimum $(\varphi$ $=2 \pi / 3$, dashed lines). More precisely, the spatial distributions consist of the moduli of acoustic pressure $\widetilde{p}(x)$ and acoustic volume velocity $\widetilde{u}(x)$, the phase shift $\Phi_{p u}(x)$ between pressure and velocity fluctuations, and the active $\mathcal{I}(x)$ and reactive $\mathcal{J}(x)$ acoustic powers plotted with respect to the $x$-coordinate [the open circles in Fig. 3(a) corresponding to the measured acoustic pressures $\left.\widetilde{p}\left(x_{i}\right)\right]$. First of all, it is noticeable that the calculation of the spatial distribution of the acoustic field is not perfectly accurate: there is no perfect matching between the calculated distributions in each of the three regions $\left(x \in\left[L_{1} ; L_{2}\right], x \in\left[L_{2} ; L_{3}\right]\right.$ and $\left.x \in\left[L_{3} ; L\right]\right)$. This mismatching appears clearly from the discontinuities of $\Phi_{p u}(x)$ at positions $x=L_{2}$ and $x=L_{3}$ (note however that the drops observed for the acoustic volume velocities at $x=L_{2}$ and $x=L_{3}$ in Fig. 3(b) are also due to the change in acoustic volume velocity induced by the auxiliary sources themselves). However, we consider that these discrepancies, 

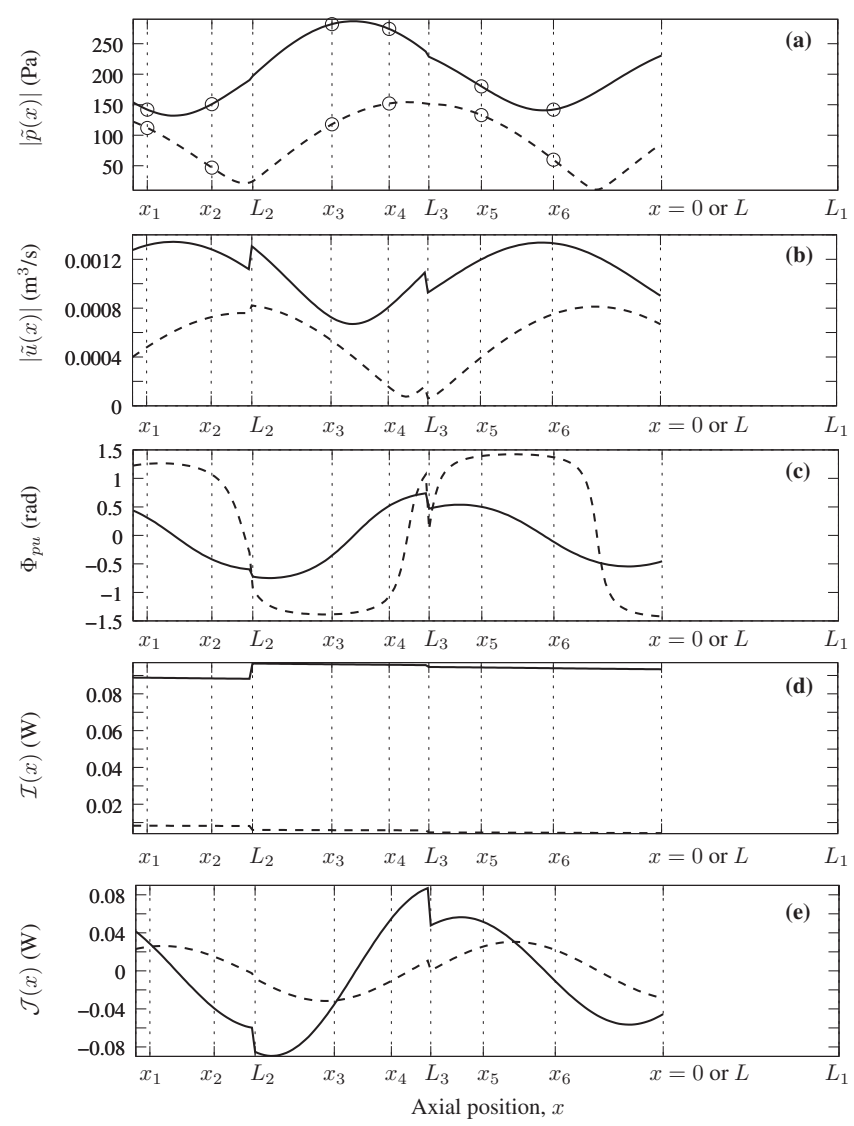

FIG. 3. Spatial distribution of the acoustic field with respect to the $x$-coordinate: (a) modulus $|\widetilde{p}(x)|$ of the acoustic pressure, (b) modulus $|\widetilde{u}(x)|$ of the acoustic volume velocity, (c) phase shift $\Phi_{p u}(x)$ between acoustic pressure and acoustic volume velocity, (d) active acoustic power $\mathcal{I}(x)$, and (e) reactive acoustic power $\mathcal{J}(x)$. The open circles correspond to the measured acoustic pressures $\widetilde{p}\left(x_{i}\right)$.

which should be attributed to some lack of accuracy inherent to the two microphone method, do not question the analysis presented hereafter. By comparing the obtained distributions for $\varphi=-2 \pi / 7$ (solid lines) and $\varphi=2 \pi / 3$ (dashed lines) in Figs. 3(a)-3(c), it appears that the phase shift $\varphi$ between the auxiliary sources strongly impacts the distribution of the acoustic field. More precisely, there are strong differences in terms of amplitudes (due to differences in thermoacoustic amplification) and in terms of positions of the extrema. Observing the distributions of the active [Fig. 3(d)] and reactive [Fig. 3(e)] acoustic powers is also instructive: in both cases, the positive sign of the active power means that there exists a traveling wave component of the acoustic wave which propagates clockwise along the waveguide. However for $\varphi$ $=2 \pi / 3$, the traveling wave component remains weak and the acoustic wave established in the waveguide is a quasi standing wave $(\mathcal{J}>\mathcal{I})$, while for $\varphi=-2 \pi / 7$ the acoustic field has an important traveling wave component $(\mathcal{I}>\mathcal{J})$. These observations indicate that the appropriate tuning of the auxiliary sources may encourage the development of a traveling acoustic wave running clockwise along the waveguide which is preferentially amplified through the thermoacoustic core (in the present case). More generally, it is clear from these results that the auxiliary sources can contribute to tune the

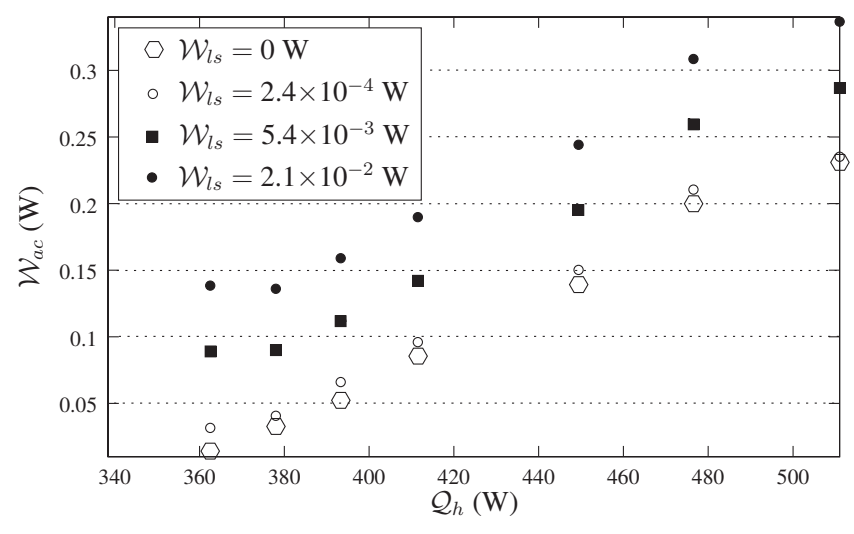

FIG. 4. Acoustic power $\mathcal{W}_{a c}$ available in the cold part of the waveguide with respect to the heating power supply $\mathcal{Q}_{h}$ for different electrical powers $\mathcal{W}_{l s}$ provided to the loudspeakers.

acoustic field in the annular waveguide in such a way that the thermoacoustic amplification occurring in the stack is optimized.

\section{EXPERIMENTAL STUDY OF THE ENGINE ABOVE THE ONSET OF THERMOACOUSTIC INSTABILITY}

In this section, the effect of the auxiliary sources is analyzed above the onset of thermoacoustic instability $\left(\mathcal{Q}_{h}\right.$ $\left.>\mathcal{Q}_{\text {onset }}\right)$. In such conditions, there exists a competition between forced oscillations (due to the auxiliary sources) and self-sustained oscillations (i.e. the thermoacoustic instability), which may lead to complicated phenomena such as synchronization or quenching. ${ }^{30}$ The aim of the experiments presented below is not to study this competition in details, but simply to examine if the auxiliary sources are able to increase significantly the acoustic power produced by thermoacoustic conversion. To do this, the following protocol has been followed in the experiments: (1) the auxiliary sources are first switched off, and the heating power $\mathcal{Q}_{h}$ is set to some value above $Q_{\text {onset }}$ leading to the onset of thermoacoustic instability; (2) after a time delay of a few minutes, necessary to reach the stabilization of both the acoustic wave amplitude and the temperature field, the steady state frequency $f_{\text {steady }}$ of thermoacoustic oscillations is accurately measured; (3) the auxiliary sources are switched on with the same electric voltage at frequency $f_{\text {steady }}$, the phase shift $\varphi$ between the input signals being tuned to $-\pi / 3$, which corresponds to the optimum phase shift observed previously just below threshold; (4) when the new steady state conditions are reached, the acoustic pressures $\widetilde{p}\left(x_{i}\right)$ are measured in order to compute the average acoustic power $\mathcal{W}_{a c}$.

In figure 4 , the measured average acoustic power $\mathcal{W}_{a c}$ is presented with respect to the heating power $\mathcal{Q}_{h}$ (ranging from 363 to $511 \mathrm{~W}$ ), and for different values of the electric power $\mathcal{W}_{l s}$ supplied to the auxiliary sources $\left(\mathcal{W}_{l s}=0 \mathrm{~W}\right.$ corresponds to the case when the sources are switched off). First of all, it appears that switching on the auxiliary sources always leads to a significant increase in the acoustic power $\mathcal{W}_{a c}$, however the heating power is fixed (from 363 to 511 $\mathrm{W})$. Even more important is the fact that the gain in acoustic power is always significantly larger than the electrical power $\mathcal{W}_{l s}$ supplied to the sources. For instance, when the heating 


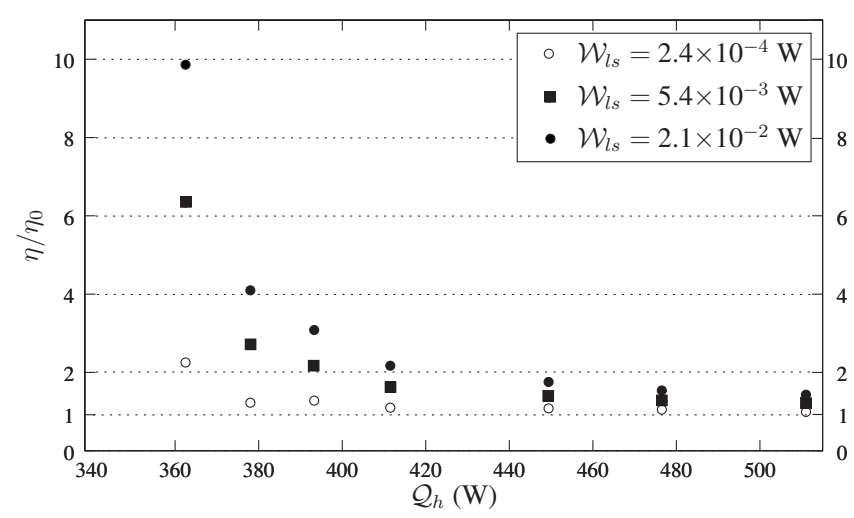

FIG. 5. Efficiency ratio $\eta / \eta_{0}$ with respect to $\mathcal{Q}_{h}$ for different electrical powers $\mathcal{W}_{l s}$ provided to the loudspeakers.

power supply $\mathcal{Q}_{h}$ is fixed to $363 \mathrm{~W}$, the increase in acoustic power $\Delta \mathcal{W}_{a c}$ is of about $1.8 \times 10^{-2} \mathrm{~W}$ for $\mathcal{W}_{l s}=2.4$ $\times 10^{-4} \mathrm{~W}, \quad \Delta \mathcal{W}_{a c} \approx 7.5 \times 10^{-2} \mathrm{~W}$ for $W_{l s}=5.4 \times 10^{-3} \mathrm{~W}$, and $\Delta \mathcal{W}_{a c} \approx 12.4 \times 10^{-2} \mathrm{~W}$ for $\mathcal{W}_{l s}=2.1 \times 10^{-2} \mathrm{~W}$. This clearly indicates that the increase in acoustic power (which is always significantly larger than $\mathcal{W}_{l s}$ ) cannot be attributed to the own contribution of the auxiliary sources to the acoustic field, but to the effective control of the acoustic field by the auxiliary sources leading to a significant increase in the thermoacoustic amplification process. It is important to note that in the experiments presented here, the temperature distribution along the thermoacoustic core has been checked before and after switching on the sources, and the resulting differences in temperature do not exceed $2 \mathrm{~K}$. This indicates that the acoustically induced variations in the temperature field $^{7,12,14}$ are relatively weak so that these variations in the temperature field may not be responsible for the large increase in the average power when turning on the auxiliary sources.

In Fig. 5, the ratio $\eta / \eta_{0}$ is presented with respect to $\mathcal{Q}_{h}$, where $\eta_{0}=\mathcal{W}_{a c} / \mathcal{Q}_{h}$ is the initial engine's efficiency without active control $\left(\mathcal{W}_{l s}=0 \mathrm{~W}\right)$ and $\eta=\mathcal{W}_{a c} /\left(\mathcal{Q}_{h}+\mathcal{W}_{l s}\right)$ is the overall engine's efficiency when the auxiliary sources are switched on. From the results presented in Fig. 5, it appears that activating the auxiliary sources (with appropriate phasing) results in a significant increase in the engine's efficiency which can reach one order of magnitude (for $\mathcal{Q}_{h}=363 \mathrm{~W}$ and $\left.\mathcal{W}_{l s}=2.1 \times 10^{-2} \mathrm{~W}, \eta / \eta_{0} \simeq 9.9\right)$. However, it also appears that this gain in efficiency gradually decreases as $\mathcal{Q}_{h}$ increases. In the present case, note that when $\mathcal{Q}_{h}=511 \mathrm{~W}$, the ratio $\eta / \eta_{0}$ approaches 1 for $W_{l s}=2.4 \times 10^{-4} \mathrm{~W}$, while the gain in efficiency is still of about $43 \%(\eta / \eta \simeq 1.43)$ for $\mathcal{W}_{l s}=2.1 \times 10^{-2} \mathrm{~W}$.

\section{DISCUSSION AND CONCLUSION}

From the experimental study presented in Sec. IV, it appears that the use of additional auxiliary sources to maximize the thermoacoustic amplification process is a promising technical solution which could lead to significant improvements of the efficiency of thermoacoustic engines. It is, however, too early to draw such a conclusion because some aspects of this study are open to criticism and some other aspects should be elucidated.
Firstly, the experimental apparatus studied here has an extremely low efficiency $\left(\eta_{0}^{\max } \approx 5 \times 10^{-4}\right)$. This low efficiency is due to various reasons, like the use of air as working fluid instead of pressurized helium, ${ }^{26}$ or like the external heating process which results in significant heat losses (only a small part of the heat input is used to impose a temperature gradient along the stack). Moreover, as mentioned earlier, the experimental apparatus studied here is not an actual engine in the sense that it is not equipped with an acoustic load. The next step in future works should be to connect the annular thermoacoustic engine to an acoustic load, as in the TASHE (Ref. 3) for which the acoustic load consists of a quarterwavelength resonator and a R-C acoustic load. ${ }^{27}$ It is however very important to note that the thermoacoustic engine described in Ref. 3 (or equivalently in Ref. 2 where the acoustic load consists of an electrodynamic alternator) is quite different from the one studied here: in particular, the operating frequency is such that the corresponding wavelength is significantly larger than the unwrapped length of the closed loop waveguide. This means that the rules adopted here for placing the auxiliary sources should be reviewed on the basis of a model describing the distribution of the acoustic field in the presence of an acoustic load.

Secondly, an important point concerning the auxiliary sources has not been studied extensively here. We have demonstrated that the acoustic coupling between the loudspeakers and the waveguide, which consists of a small diameter cylindrical hole, must be realized in order to prevent from a large increase in the heat input $Q_{\text {onset }}$ necessary for the onset of the thermoacoustic instability. We have also demonstrated that the efficiency of the active control depends on the electric power $\mathcal{W}_{l s}$ supplied to the loudspeakers relative to the heating power supply $\mathcal{Q}_{h}$. However, the choice of enclosed electrodynamic loudspeakers as auxiliary sources has been guided here by convenience, and there are probably better choices concerning the electromechanical parameters of the sources, the kind of electroacoustic actuation (electrodynamic, piezoelectric, etc.) or the way of coupling the transducers to the waveguide. It is thus important to clarify these points before trying to add auxiliary sources in a high power, high efficiency thermoacoustic engine. In such an engine, the fluid is indeed highly pressurized so that the amplitudes of pressure oscillations can reach several bars: the problem then consists in finding appropriate acoustic sources able to control the high amplitude self-sustained thermoacoustic oscillations, these sources being supplied by an electrical power $\mathcal{W}_{l s}$ greatly below the heat input $\mathcal{Q}_{h}$ supplied for the thermoacoustic amplification.

Thirdly, it is worth noting that, within the framework of this study, the experiments has been carried out only for $\varphi$ $=-\pi / 3$ above the onset, because this study primarily intends to prove the concept of active control. It is however clear that the optimum phase $\varphi$ may change with $\mathcal{Q}_{h}$ above the onset of thermoacoustic instability, and that complementary measurements should be carried out to investigate if a more precise control of $\varphi$ can lead to a significant increase in the engines efficiency. 
Future works will be devoted to complementary experimental and theoretical studies aiming at clarifying the aspects mentioned above.

\section{ACKNOWLEDGMENTS}

This work has been supported by the French National Research Agency (Contract No. ANR-05-BLANC-0016-2).

${ }^{1}$ G. W. Swift, J. Acoust. Soc. Am. 84, 1145 (1988).

${ }^{2}$ S. Backhaus, E. Tward, and M. Petach, Appl. Phys. Lett. 85, 1085 (2004).

${ }^{3}$ S. Backhaus G.W. Swift, Nature (London) 399, 335 (1999)

${ }^{4}$ H. Yuan, S. Karpov, and A. A. Prosperetti, J. Acoust. Soc. Am. 102, 3497 (1997).

${ }^{5}$ V. Gusev, H. Bailliet, P. Lotton, and M. Bruneau, Acust. Acta Acust. 86, 25 (2000).

${ }^{6}$ R. S. Wakeland and R. M. Keolian, J. Acoust. Soc. Am. 115, 2071 (2004).

${ }^{7}$ T. Yazaki, A. Tominaga, and Y. Narahara, J. Heat Transfer 105, 889 (1983).

${ }^{8}$ L. Rayleigh, Philos. Trans. R. Soc. London, Ser. A 36, 10 (1883).

${ }^{9}$ D. C. Gedeon, Cryocoolers 9, 385 (1997).

${ }^{10}$ N. Rott, Z. Angew. Math. Phys. 25, 417 (1974).

${ }^{11}$ M. F. Hamilton, Y. A. Ilinskii, and E. A. Zabolotskaya, J. Acoust. Soc. Am. 114, 3092 (2003).

${ }^{12}$ V. Gusev, S. Job, H. Bailliet, P. Lotton, and M. Bruneau, J. Acoust. Soc. Am. 108, 934 (2000).

${ }^{13}$ H. Bailliet, V. Gusev, R. Raspet, and R. Hiller, J. Acoust. Soc. Am. 110,
1808 (2001).

${ }^{14}$ M. Mironov, V. Gusev, Y. Auregan, P. Lotton, and M. Bruneau, J. Acoust. Soc. Am. 112, 441 (2002).

${ }^{15}$ M. F. Hamilton, Y. A. Ilinskii, and E. A. Zabolotskaya, J. Acoust. Soc. Am. 111, 2076 (2002).

${ }^{16}$ G. Penelet, V. Gusev, P. Lotton, and M. Bruneau, Phys. Rev. E 72, 016625 (2005).

${ }^{17}$ G. Penelet, V. Gusev, P. Lotton, and M. Bruneau, Phys. Lett. A 351, 268 (2006).

${ }^{18}$ A. T. A. M. de Waele, J. Sound Vib. 325, 974 (2009).

${ }^{19}$ W. C. Ward and G. W. Swift, J. Acoust. Soc. Am. 95, 3671 (1994).

${ }^{20}$ J. R. Olson and G. W. Swift, Cryogenics 37, 769 (1997).

${ }^{21}$ G. W. Swift, D. L. Gardner, and S. Backhaus, J. Acoust. Soc. Am. 105, 711 (1999).

${ }^{22}$ G. Penelet, S. Job, V. Gusev, P. Lotton, and M. Bruneau, Acust. Acta Acust. 91, 567 (2005).

${ }^{23}$ C. Desjouy, G. Penelet, P. Lotton, and J. Blondeau, J. Acoust. Soc. Am. 126, 2176 (2009).

${ }^{24}$ P. A. Ceperley, Am. J. Phys. 60, 938 (1992).

${ }^{25}$ H. Bodén and M. Abom, J. Acoust. Soc. Am. 79, 541 (1986).

${ }^{26}$ G. W. Swift, Thermoacoustics: A Unifying Perspective for Some Engines and Refrigerators (Acoustical Society of America, Sewickley, 2002).

${ }^{27}$ A. M. Fusco, W. C. Ward, and G. W. Swift, J. Acoust. Soc. Am. 91, 2229 (1992).

${ }^{28}$ G. Krishnappa, J. Acoust. Soc. Am. 69, 307 (1981).

${ }^{29}$ A. Atchley, J. Acoust. Soc. Am. 92, 2907 (1992).

${ }^{30}$ A. H. Nayfeh and D. T. Mook, Nonlinear Oscillations (Wiley, New York, 1979). 